\title{
Compilation de 3751 dosages sanguins de stupéfiants obtenus par 19 experts, dans le cadre de l'application de la loi Gayssot
}

\section{Compilation of 3751 quantitative drug deter- minations in blood obtained by 19 experts, in the framework of the enforcement of the Gayssot Act}

Gilbert PÉPIN*(1), Gaëlle DUFFORT ${ }^{(1)}$, Nathalie ROMMEL(1), Pascal KINTZ, Véronique DUMESTRE-TOULET, Marie-France KERGUERIS, Gérard LACHATRE, Mustapha MOULSMA, Jean-Pierre GOULLÉ, Christian LACROIX, Ivan RICORDEL, Patrick MURA, Françoise VINCENT, Alain GRUSON, Anne GRUSON, Michel LHERMITTE, Bernard CAPOLAGHI, Alain TURCANT, Marie-Hélène GHYSEL, Philippe CORTEEL

(1) Laboratoire d'Expertises TOXLAB, 7, rue Jacques Cartier - 75018 PARIS

* Auteur à qui adresser la correspondance : Gilbert PÉPIN, Laboratoire d'Expertises TOXLAB,

7, rue Jacques Cartier - 75018 PARIS - Tél : 0158592800 - Fax 0158592801 - E-mail : toxlab@wanadoo.fr

(Reçu le 3 avril 2003 ; accepté le 26 avril 2003)

\section{RÉSUMÉ}

Cet article présente une compilation de 3751 dosages sanguins, réalisés auprès de conducteurs impliqués dans un accident mortel de la circulation, ainsi que le prévoit la loi Gayssot du 18 juin 1999 et son décret d'application du 27 août 2001, loi relative à la sécurité routière et traitant de l'implication des stupéfiants dans les accidents mortels de la circulation.

Il résulte de cette compilation statistique que tous âges confondus, $17 \%$ des analyses sanguines réalisées sur des conducteurs impliqués dans un accident mortel de la circulation, mettaient en évidence la présence de stupéfiants, dont $13,8 \%$ étaient positives au $\Delta$-9-tétrahydrocannabinol (THC), principe actif stupéfiant hallucinogène du cannabis. Dans la population des conducteurs de moins de 27 ans,

\section{SUMMARY}

This paper presents a compendium of 3751 blood analyses, obtained from drivers involved in a fatal car crash, as requested by the French law of June 18, 1999 (so called Gayssot Act) and its application order from August 27, 2001. From the whole population, $17 \%$ were positive for at least one drug of abuse, including $13.8 \%$ positive for cannabis. With respect to the population below 27 year-old, $29.8 \%$ were positive for at least one drug of abuse, including $27.2 \%$ positive for cannabis. A combination ethanol and cannabis was observed in $25 \%$ of the cases. In case of a positive result for any drug of abuse, the law requests testing for psychoactive pharmaceuticals. Such combinations were rather infrequent. 
$29,8 \%$ des analyses sanguines mettaient en évidence la présence de stupéfiants dont 27,2\% étaient positives aux cannabinoïdes. Par ailleurs l'alcool était également présent dans $25 \%$ des analyses positives au cannabis. Enfin en cas de résultat positif aux stupéfiants la loi prévoyait la recherche de médicaments psychoactifs, cette association présente une incidence relativement faible.

Cette étude a mis en évidence une importante proportion de conducteurs sous l'influence de stupéfiants impliqués dans des accidents graves ou mortels, confirmant le fait que les effets neuropsychiques des stupéfiants sont un facteur potentiel important d'accidents.

\section{MOTS-CLÉS}

Loi Gayssot, dosages sanguins, stupéfiants, cannabis, conduite automobile, risques d'accidents.

\section{Introduction}

Alors que la consommation d'alcool au volant en France est sanctionnée par la loi depuis plus de 30 ans et régie par un seuil limite depuis le 8 décembre 1983, un vide juridique a longtemps demeuré, autour de la conduite sous l'influence de produits stupéfiants. Pourtant les effets neuropsychiques de ces substances étaient bien connus ( 1 - 4) et laissaient craindre une augmentation du risque d'accidents de la route. Ainsi la Société Française de Toxicologie Analytique (SFTA) a créé, en 1995, une commission «drogues et conduite automobile» dont l'un des objectifs a été d'étudier la prévalence de l'usage de stupéfiants chez les conducteurs impliqués dans des accidents corporels ou mortels de la circulation.

* En 1998, P. Marquet et coll. ont réalisé une étude multicentrique sur 296 conducteurs impliqués dans un accident corporel de la voie publique et 278 témoins (5). Les urines ont été analysées par FPIA puis CPG$\mathrm{SM}$ et les résultats obtenus sont consignés dans le tableau I. La comparaison entre les conducteurs et les témoins a mis en évidence une implication importante des cannabinoïdes chez les conducteurs impliqués dans un accident corporel.

* Une étude a été réalisée en 1998 par G. Pépin et 7 experts toxicologues agréés par différentes cours d'Appel en France (6). Cette étude concernait la

Tableau I : Résultats de l'étude multicentrique menée par P. Marquet et coll. en 1998 mesurant la prévalence des stupéfiants sur 296 conducteurs impliqués dans un accident corporel et 278 témoins (5).

\begin{tabular}{|c|c|c|}
\hline & Conducteurs & Témoins \\
\hline Cannabis & $13,9 \%$ & $7,5 \%$ \\
\hline Opiacés & $10,5 \%$ & $10,4 \%$ \\
\hline Amphétamines & $1,4 \%$ & $2,5 \%$ \\
\hline Cocaïne & $1 \%$ & $1,1 \%$ \\
\hline
\end{tabular}

This study revealed that many drivers involved in serious or fatal accidents were under the influence of drugs of abuse and it confirms that their neuropsychic effects can be considered as an important risk of road crash.

\section{KEY-WORDS}

Gayssot Act, blood determination, drugs of abuse, cannabis, driving, road crash risk.

recherche de stupéfiants dans le sang de conducteurs, pour lesquels la prise de stupéfiants pouvait être suspectée, conducteurs impliqués dans des accidents corporels graves ou mortels sur réquisition d'officiers de police judiciaire et de procureurs de la République ; les résultats regroupés dans le tableau II, montrent que sur 94 échantillons analysés, $56,4 \%$ contenaient au moins un des quatre stupéfiants (cannabis, opiacés, cocaïne, amphétamines), dont $34 \%$ pour le cannabis. D'autre part, 39,1\% des échantillons positifs aux stupéfiants ou aux médicaments ne contenaient pas d'alcool.

* L'étude multicentrique publiée en 1999 par G. Pépin et coll. portant sur 164 conducteurs impliqués dans un accident grave ou mortel (6) a révélé grâce à des analyses sanguines par chromatographie en phase gazeuse couplée à la spectrométrie de masse (CPG/SM), une prévalence importante du cannabis ( $16 \%$ des conducteurs). Ces résultats sont détaillés dans le tableau III.

Tableau II : Résultats sanguins de l'étude réalisée en 1998 sur 94 expertises toxicologiques chez des conducteurs suspectés d'être sous l'influence de stupéfiants lors d'accident grave ou mortel (6).

\begin{tabular}{|c|c|c|}
\hline & Nombre de sujets positifs & Prévalence (\%) \\
\hline Cannabis & 32 & 34 \\
\hline Opiacés & 15 & 16 \\
\hline Amphétamines & 4 & 4,2 \\
\hline Cocaïniques & 2 & 2,1 \\
\hline
\end{tabular}

Tableau III : Résultats sanguins de l'étude réalisée en 1999 sur 164 expertises toxicologiques réalisées chez des conducteurs impliqués dans un accident grave ou mortel (7).

\begin{tabular}{|c|c|c|}
\hline & Nombre de sujets positifs & Prévalence (\%) \\
\hline Cannabis & 26 & 16 \\
\hline Opiacés & 5 & 3 \\
\hline Amphétamines & 1 & 0,7 \\
\hline Cocaïniques & 0 & 0 \\
\hline
\end{tabular}


* Une étude de P. Kintz et coll. datant de 2000 menée dans la région de Strasbourg a confirmé cette tendance puisque sur 198 conducteurs impliqués dans un accident corporel et hospitalisés, $11 \%$ des conducteurs avait une alcoolémie supérieure à $0,5 \mathrm{~g} / \mathrm{l}, 10 \%$ des conducteurs étaient positifs au cannabis, $2 \%$ aux opiacés, $0,5 \%$ à la cocaïne et $0,5 \%$ étaient positifs à l'ecstasy tandis que $6 \%$ avaient des benzodiazépines dans le sang (7).

* Enfin une vaste étude multicentrique dans le cadre d'un Programme Hospitalier de Recherche Clinique (P.H.R.C.) a été réalisée en 2000 et 2001 en France par Mura et coll., sur 900 conducteurs impliqués dans un accident corporel et 900 sujets témoins. Le protocole de cette étude a été soumis à différents comités scientifiques de la Direction Générale de la Santé (D.G.S.) et accepté par des experts spécialisés en accidentologie $(8,9)$. Elle a montré que lorsque des conducteurs de moins de 27 ans ont consommé du cannabis, la fréquence des accidents est multipliée par 2,5. Dans cette tranche d'âge, $20 \%$ des conducteurs impliqués dans un accident corporel avaient du THC dans le sang versus $9 \%$ chez les témoins et chez les conducteurs ayant du cannabis et de l'alcool dans le sang, la fréquence d'accidents était multipliée par 4,6.

Ces premières études tendaient déjà à mettre en évidence le rôle important de l'influence des stupéfiants dans les accidents mortels ou corporels graves de la circulation. La parution de la loi «Gayssot» (Loi ${ }^{\circ}{ }^{99}$ -
505 du 18 juin 1999) relative à la sécurité routière devait permettre de réaliser, en France, pour l'année 2004, une étude épidémiologique à grande échelle incluant outre le rôle des stupéfiants, également l'incidence de l'alcoolémie et des médicaments psychotropes dans les accidents mortels de la circulation. Le décret $n^{\circ} 2003-293$ du 31 mars 2003 paru le $1^{\text {er }}$ avril 2003 n'a pas intégré cette étude épidémiologique.

Un an après la parution du décret d'application $n^{\circ}$ 2001-751 du 27 août 2001 définissant les conditions de la recherche de stupéfiants pratiquée sur les conducteurs impliqués dans un accident mortel de la circulation, il semblait intéressant de compiler les résultats statistiques de l'ensemble des 40 laboratoires qui s'étaient inscrits auprès de la préfecture de Police pour ce faire et dont les noms figurent entre autres sur le site internet du ministère de la justice : www.justice.gouv.fr La commission «drogues et conduite automobile» de la SFTA a mis en place pour ces 40 laboratoires un contrôle qualité externe qui consistait à mettre en évidence et à doser 7 molécules appartenant aux quatre familles de stupéfiants (cannabinoïdes, opiacés, cocaïniques, amphétaminiques) incluant leurs métabolites.

Sur cette base, seuls les résultats des 19 laboratoires ayant obtenu des résultats satisfaisants à ce contrôle qualité ont pu être pris en considération, réunissant 3751 résultats d'analyses sanguines (10) et sont présentés dans le tableau IV.

Tableau IV : Recherche de stupéfiants en application de la loi Gayssot : 3751 analyses sanguines réalisées par 19 experts en 2001.

\begin{tabular}{|c|c|c|c|c|c|}
\hline \multicolumn{2}{|c|}{ Type de stupéfiants } & \multicolumn{2}{|c|}{ Totaux } & \multicolumn{2}{|c|}{ \% moyen des laboratoires } \\
\hline \multirow{2}{*}{$\mathrm{THC}+\mathrm{THC}-\mathrm{COOH}$} & Seuls & 474 & \multirow{2}{*}{514} & $12,6 \%(5,9-15,4)$ & \multirow{2}{*}{$13,8 \%$} \\
\hline & Associé & 40 & & $1,2 \%(0,4-2,2)$ & \\
\hline \multirow{2}{*}{$\begin{array}{c}\text { THC-COOH seul } \\
(\text { nombre de réponses }=2550)\end{array}$} & Seul & 124 & \multirow{2}{*}{146} & $4,9 \%(2-10,7)$ & \multirow{2}{*}{$5,8 \%$} \\
\hline & Associé & 22 & & $0,9 \%(0,6-3)$ & \\
\hline \multirow{2}{*}{ OPIACÉS } & Seul & 112 & \multirow{2}{*}{132} & $3 \%(0,4-7,3)$ & \multirow{2}{*}{$3,6 \%$} \\
\hline & Associé & 20 & & $0,6 \%(0-5,3)$ & \\
\hline \multirow{2}{*}{ COCAINIQUES } & Seul & 15 & \multirow{2}{*}{28} & $0,4 \%(0-2,6)$ & \multirow{2}{*}{$0,8 \%$} \\
\hline & Associé & 13 & & $0,4 \%(0-1,3)$ & \\
\hline \multirow{2}{*}{ AMPHÉTAMINIQUES } & Seul & 35 & \multirow{2}{*}{62} & $0,9 \%(0-2,7)$ & \multirow{2}{*}{$1,7 \%$} \\
\hline & Associé & 27 & & $\mathbf{0 , 8} \%(0-2,2)$ & \\
\hline $\begin{array}{c}\text { TOTAL } \\
\text { Stupéfiants positifs }\end{array}$ & $\begin{array}{c}\text { Sans } \\
\text { association } \\
\text { avec autres } \\
\text { stupéfiants }\end{array}$ & \multicolumn{2}{|c|}{637} & \multicolumn{2}{|l|}{$17 \%$} \\
\hline
\end{tabular}


Ces chiffres montrent une prévalence importante $(17 \%)$ de la présence de stupéfiants dans le sang des conducteurs impliqués dans un accident grave ou mortel. De plus, cette observation sur plusieurs milliers de résultats confirme les conclusions des travaux précédents sur le sujet. Le cannabis est le stupéfiant le plus fréquemment rencontré : dans $13,8 \%$ des cas, le delta9-tétrahydrocannabinol (THC) et/ou l'acide delta-9tétrahydrocannabinol carboxylique (THC-COOH) étaient présents seuls ou associés à d'autres stupéfiants.

\section{Seuils de positivité : difficul- tés rencontrées pour le THC}

Le seuil de positivité au THC que nous avons retenu pour notre étude est de $1 \mathrm{ng} / \mathrm{ml}$ de sang car il correspond au seuil défini par l'article 11 de l'arrêté du 5 septembre 2001.

L'étude des 44 cas positifs au THC et THC-COOH relevés au laboratoire TOXLAB (Figure 1) montre qu'un grand nombre de cas ne sont pas pris en compte dans les résultats de notre étude statistique, du fait que la concentration en THC était inférieure à $1 \mathrm{ng} / \mathrm{ml}$ (seuil légal de positivité).

Il existe de réelles difficultés pour établir une relation entre la concentration sanguine en THC et ses effets au niveau cérébral. En l'état actuel de nos connaissances, il est considéré que la seule présence de THC dans le sang permet d'estimer que le sujet était sous influence de cannabis au moment du prélèvement (11).

C'est pourquoi, certains auteurs ont estimé que le seuil légal actuel de $1 \mathrm{ng} / \mathrm{ml}$ pouvait être ramené au seuil analytique de $0,5 \mathrm{ng} / \mathrm{ml}$, voire $0,2 \mathrm{ng} / \mathrm{ml}$ (12).

En outre, comment envisager un seuil légal de cannabis comme c'est le cas pour l'alcool, produit dont la consommation est licite, alors que le cannabis est un produit stupéfiant dont la consommation est illicite?

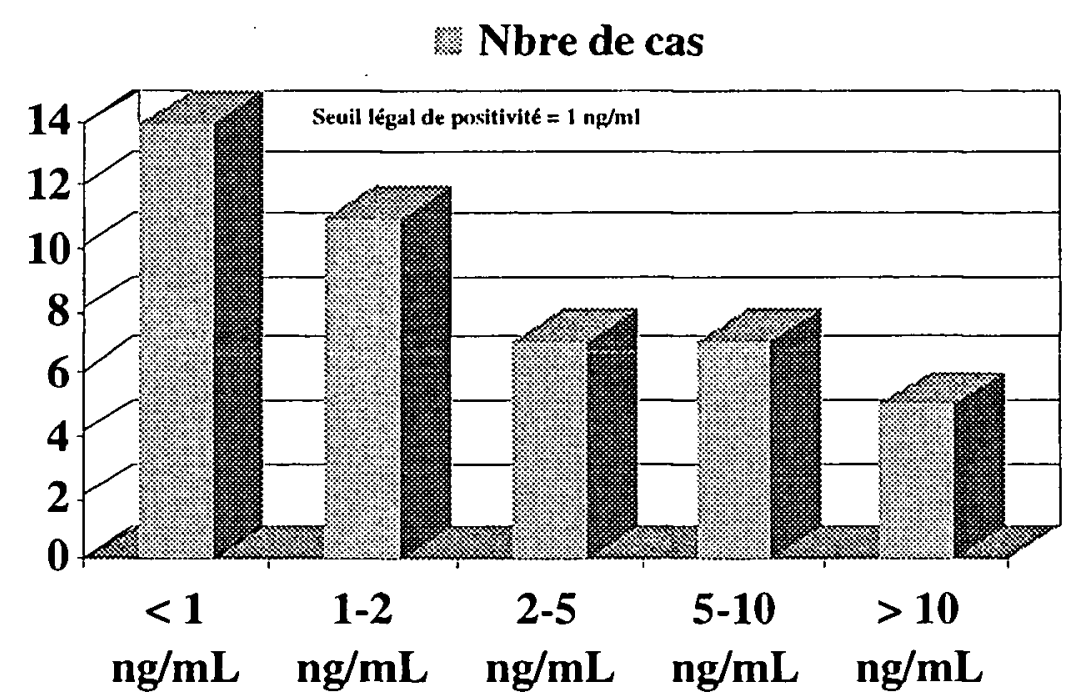

Figure 1: Concentration de THC des 44 cas positifs au cannabis relevés à TOXLAB.

\section{Cannabis : prévalence chez les jeunes conducteurs}

Les accidents de la route représentant en France la première cause de mortalité chez les jeunes, nous nous sommes intéressés plus particulièrement à la population des sujets de moins de 27 ans. Les analyses réalisées dans cette tranche d'âge ont mis en évidence du THC et/ou du THC-COOH dans $27,2 \%$ des cas versus $13,8 \%$ toutes tranches d'âge confondues (Tableau V).

De plus $29,8 \%$ des analyses réalisées chez les moins de 27 ans étaient positives à l'un des quatre stupéfiants contre $17 \%$ pour l'ensemble des conducteurs impliqués dans un accident grave ou mortel de la circulation.

Parmi les 44 cas positifs au THC et THC-COOH relevés au laboratoire TOXLAB, la tranche d'âge la plus représentée concernait les $20-27$ ans (55,8\%), suivie des 18-20 ans (21\%).

Ces statistiques corrèlent parfaitement celles obtenues dans le cadre de l'étude de P. Mura et coll. $(8,9)$ qui soulignaient également une prévalence importante du THC chez les moins de 27 ans.

\section{Stupéfiants et alcool}

Parmi les 19 experts participant à l'étude, 3 d'entre eux ont pu fournir des données concernant la présence concomitante d'alcool et de stupéfiants, représentant 236 analyses sanguines positives aux stupéfiants. On constate que dans $21,6 \%$ des cas, de l'alcool était également présent en plus d'une substance stupéfiante. Le tableau VI montre que la prévalence de l'alcool en association avec un stupéfiant est à peu près équivalente pour les cannabinoïdes, les cocaïniques et les amphétaminiques (environ $25 \%$ ), mais qu'elle est bien plus faible avec les opiacés $(7,7 \%)$.

\section{Stupéfiants et médicaments}

Différentes études épidémiologiques françaises et étrangères (13-17) semblent avoir démontré le rôle de certains médicaments psychotropes dans les accidents de la circulation (benzodiazépines, antidépresseurs imipraminiques sédatifs..).

Dans le décret $n^{\circ} 2001-751$ du 27 août 2001 relatif à la recherche de stupéfiants pratiquée sur les conducteurs impliqués dans un accident mortel de la circulation, la recherche de médicaments psychoactifs était également obligatoire lorsque des stupéfiants étaient détectés dans le sang.

Nous avons effectué une synthèse de 448 résultats obtenus suite à la recherche de médicaments psychoactifs 
Tableau $V:$ Recherche de stupéfiants sur de jeunes conducteurs de moins de 27 ans en application de la loi Gayssot: 1150 analyses sanguines réalisées par 19 experts en 2001.

\begin{tabular}{|c|c|c|c|c|c|}
\hline \multicolumn{2}{|c|}{ Type de stupéfiants } & \multicolumn{2}{|c|}{ Totaux } & \multicolumn{2}{|c|}{$\%$ moyen des laboratoires } \\
\hline \multirow{2}{*}{ THC + THC-COOH } & Seuls & 287 & \multirow{2}{*}{314} & $25 \%(15,9-44,8)$ & \multirow{2}{*}{$27,2 \%$} \\
\hline & Associé & 27 & & $2,3 \%(1,1-5,2)$ & \\
\hline \multirow{2}{*}{$\begin{array}{l}\text { THC-COOH seul } \\
\text { (nombre de réponses = 589) }\end{array}$} & Seul & 50 & \multirow{2}{*}{58} & $\mathbf{8 , 5} \%(1,6-9)$ & \multirow{2}{*}{$9,9 \%$} \\
\hline & Associé & 8 & & $1,4 \%(0,6-2)$ & \\
\hline \multirow{2}{*}{ OPIACÉS } & Seul & 26 & \multirow{2}{*}{32} & $2,3 \%(0-9,9)$ & \multirow{2}{*}{$2,8 \%$} \\
\hline & Associé & 6 & & $\mathbf{0 , 5} \%(0-1,8)$ & \\
\hline \multirow{2}{*}{ COCAINIQUES } & Seul & 7 & \multirow{2}{*}{12} & $\mathbf{0 , 6} \%(0-3)$ & \multirow{2}{*}{$1 \%$} \\
\hline & Associé & 5 & & $\mathbf{0 , 4} \%(0-3)$ & \\
\hline \multirow{2}{*}{ AMPHÉTAMINIQUES } & Seul & 23 & \multirow{2}{*}{44} & $2,0 \%(0-6,3)$ & \multirow{2}{*}{$3,8 \%$} \\
\hline & Associé & 21 & & $\mathbf{1 , 8} \%(0-5,2)$ & \\
\hline $\begin{array}{c}\text { TOTAL } \\
\text { Stupéfiants positifs }\end{array}$ & $\begin{array}{l}\text { Sans } \\
\text { association } \\
\text { avec autres } \\
\text { stupéfiants }\end{array}$ & \multicolumn{2}{|c|}{343} & \multicolumn{2}{|l|}{$29,8 \%$} \\
\hline
\end{tabular}

Tableau VI : Prévalence de la présence d'alcool en cas d'analyses positives aux stupéfiants (étude de 236 analyses).

\begin{tabular}{|c|c|}
\hline Type de stupéfiant & $\begin{array}{c}\text { Prévalence de l'alcool lors de la présence } \\
\text { de stupéfiants }\end{array}$ \\
\hline THC + THC-COOH & $25 \%$ \\
\hline THC-COOH seul & $22,4 \%$ \\
\hline Opiacés & $7,7 \%$ \\
\hline Cocaïniques & $28,6 \%$ \\
\hline Amphétaminiques & $23,1 \%$ \\
\hline
\end{tabular}

après une recherche de stupéfiants positive. Sur ces 448 analyses positives aux stupéfiants, $27(6 \%)$ révélaient la présence de médicaments psychoactifs. Au sein de la tranche d'âge des moins de 27 ans, seulement 9 analyses $(3,8 \%)$ se sont révélées positives aux médicaments psychotropes associés aux stupéfiants.

Nous avons également effectué un bilan sur la recherche de médicaments psychoactifs après une recherche de stupéfiants positive au THC. Sur les 240 réponses des différents laboratoires participants à l'étude, tous âges confondus, 12 analyses (5\%) mettaient en évidence une association de psychotropes et de cannabis. Dans la tranche d'âge des moins de 27 ans, seulement 5 analyses $(3,2 \%)$ se sont révélées positives à la fois aux psychotropes et au cannabis.

Ces résultats sont concordants avec le fait que la population des moins de 27 ans consomme en moyenne moins de psychotropes que la majorité de la population tous âges confondus.

\section{Pourquoi des dosages san- guins ?}

Le décret $n^{\circ}$ 2001-751 du 27 août 2001 relatif à la recherche de stupéfiants pratiquée sur les conducteurs impliqués dans un accident mortel de la circulation prévoit une recherche des stupéfiants dans le sang. Ce milieu biologique a été retenu comme étant le plus approprié car c'est celui qui permet la meilleure interprétation du degré d'influence du produit sur le comportement : en effet il existe, (en dépit de quelques variations individuelles), une relation entre la quantité de stupéfiant absorbée et le taux sanguin. De plus, le dosage sanguin matérialise de façon indiscutable une consommation récente de stupéfiants et le sang n'est pas adultérable contrairement à l'urine (18).

Par ailleurs, les autres matrices biologiques (salive, sueur) sont inadaptées à la recherche de cannabis, soit concernant la sueur pour des raisons pharmacologiques (variations selon l'hygiène corporelle individuelle), soit concernant la salive pour des raisons de pollution par les fumées aboutissant à de nombreux faux positifs chez les fumeurs passifs. 


\section{Modalités opératoires du prélèvement sanguin}

L'arrêté du 5 septembre 2001 fixant les modalités du dépistage des stupéfiants prévu par le décret $n^{\circ} 2001$ 751 du 27 août 2001 indique qu'en cas d'accident mortel de la circulation, un dépistage de stupéfiants doit être réalisé par un docteur en médecine, un interne ou un étudiant en médecine autorisé et requis par un officier de police judiciaire (OPJ).

Si le dépistage urinaire s'avère positif, un prélèvement sanguin de confirmation sera alors effectué pour la recherche d'alcool, de stupéfiants et de médicaments en cas de positivité aux stupéfiants.

Ces modalités opératoires sont décrites dans la figure 2 .

\section{Durée de détectabilité dans le sang et l'urine des 4 prin- cipaux stupéfiants}

Les durées de détection ainsi que les seuils légaux et analytiques de détection des quatre principaux types de stupéfiants sont présentés dans les tableaux VII, VIII, IX et X.
Il apparaît que des molécules telles que le THC, l'héroïne, la morphine ainsi que la codéine ne sont détectables dans le sang que de quelques minutes pour les unes (héroïne) à quelques heures pour les autres. Toutefois leur métabolites comme le THC-COOH, la morphine, la benzoylecgonine (l'un des métabolites principaux de la cocaïne) ainsi que l'amphétamine ou l'ecstasy sont présents plus longtemps dans le sang (de 6 à 48 heures pour l'ensemble des molécules), ce qui peut permettre de mettre en évidence leur consommation.

\section{Stabilité des molécules stu- péfiantes et de leurs métabo- lites dans les prélèvements}

Afin de fournir une interprétation fiable des résultats et afin de s'assurer que les résultats d'analyses sanguines négatifs n'étaient pas dus à une dégradation des molécules au sein du prélèvement, nous avons effectué une recherche bibliographique concernant la stabilité des stupéfiants et de leurs métabolites dans un prélèvement conservé à $+4^{\circ} \mathrm{C}$. En ce qui concerne les opiacés, la 6-monoacétyl-morphine (principal métabolite de l'héroïne) est instable tandis que la morphine est stable à $+4^{\circ} \mathrm{C}$ des mois, voire des années.

\section{Conducteurs impliqués dans un Accident mortel} (Arrêté du 05/09/01)

Docteur en médecine, Interne des hôpitaux, Etudiant en médecine autorisé à exercer
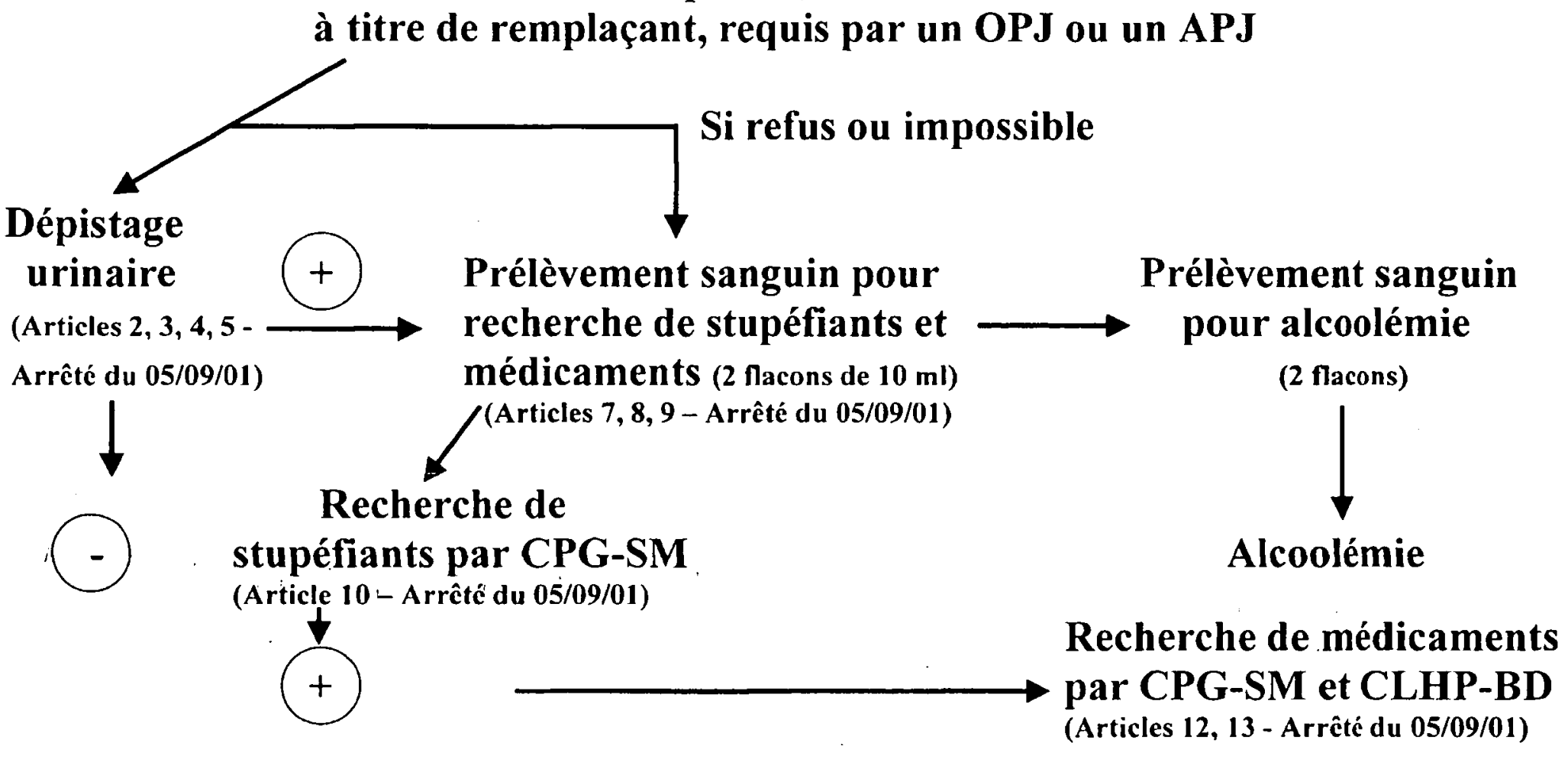

Figure 2 : Modalités opératoires du prélèvement (comment, par qui et dans quelles conditions est-il effectué). 
Annales de Toxicologie Analytique, vol. XV, n 2, 2003

Tableau VII : Durées et seuils de détection des cannabinö̈des dans le sang et l'urine.

\begin{tabular}{|c|c|c|c|}
\hline & Temps de demi-vie & Détection dans le sang & Détection dans l'urine \\
\hline THC & 14 à $36 \mathrm{~h}$ & 2 à $8 \mathrm{~h}$ & Non détecté \\
\hline THC-COOH & 24 à $72 \mathrm{~h}$ & 6 à $48 \mathrm{~h} *$ & 1 à 70 jours \\
\hline Seuil de détection légal (art 11 arrêté du $05 / 09 / 01)$ & THC $: 1 \mathrm{ng} / \mathrm{ml}$ & THC : $50 \mathrm{ng} / \mathrm{ml}$ \\
\hline Seuil de détection analytique par CPG/SM & THC $: 0,2 \mathrm{ng} / \mathrm{ml}$ & - \\
\hline
\end{tabular}

* très variable selon les individus et la fréquence de leur consommation.

Tableau VIII : Durées et seuils de détection des opiacés dans le sang et l'urine.

\begin{tabular}{|c|c|c|c|}
\hline & Temps de demi-vie & Détection dans le sang & Détection dans l'urine \\
\hline Héroïne & 0,05 à $0,15 \mathrm{~h}$ & $5 \mathrm{~min}$ & 1 à $3 \mathrm{~h}$ \\
\hline 6-Acétyl morphine & $\therefore \quad \therefore \quad 0 ; 6 \mathrm{~h}$ & 1 à $2 \mathrm{~h}$ & 2 à $8 \mathrm{~h}$ \\
\hline Morphine & 1,9 à $3,1 \mathrm{~h}$ & 6 à $12 \mathrm{~h}$ & $1 \mathrm{à} 2 \mathrm{j}$ \\
\hline Codéine & $2,4 \mathrm{~h}$ & $8 \mathrm{~h}$ & 24 à $48 \mathrm{~h}$ \\
\hline Phocodine & $45 \mathrm{~h}$ & 48 à $72 \mathrm{~h}$ & 11 à $20 \mathrm{j}$ \\
\hline \multicolumn{2}{|c|}{ Seuil de détection légal (art 11 arrêté du 05/09/01) } & $20 \mathrm{ng} / \mathrm{ml}$ & $300 \mathrm{ng} / \mathrm{ml}$ \\
\hline \multicolumn{2}{|c|}{ Seuil de détection analytique des opiacés (CPG/SM) } & 5 à $10 \mathrm{ng} / \mathrm{ml}$ & - \\
\hline
\end{tabular}

Tableau IX : Durées et seuils de détection des cocaïniques dans le sang et l'urine.

\begin{tabular}{|c|c|c|c|}
\hline & Temps de demi-vie & Détection dans le sang & Détection dans l'urine \\
\hline Cocaïne & 0,7 à $1,5 \mathrm{~h}$ & $1 \mathrm{à} 12 \mathrm{~h}$ & $5 \mathrm{~h}$ \\
\hline Benzylecgonine & 5 à $7 \mathrm{~h}$ & $12 \mathrm{à} 24 \mathrm{~h}$ & 2 à $3 \mathrm{j}$ \\
\hline Seuil de détection légal (art 11 arrêté du $05 / 09 / 01)$ & $50 \mathrm{ng} / \mathrm{ml}$ & $300 \mathrm{ng} / \mathrm{ml}$ \\
\hline Seuil de détection analytique des cocaïniques (CPG/SM) & 5 à $10 \mathrm{ng} / \mathrm{ml}$ & - \\
\hline
\end{tabular}

Tableau X : Durées et seuils de détection des amphétaminiques dans le sang et l'urine.

\begin{tabular}{|c|c|c|c|}
\hline & Temps de demi-vie & Détection dans le sang & Détection dans l'urine \\
\hline Amphétamine & 7 à $34 \mathrm{~h}$ & 12 à $24 \mathrm{~h}$ & 1 à $3 \mathrm{j}$ \\
\hline Ecstasy & 7 à $12 \mathrm{~h}$ & 6 à $12 \mathrm{~h}$ & 1 à $3 \mathrm{j}$ \\
\hline Seuil de détection légal (art 11 arrêté du $05 / 09 / 01)$ & $50 \mathrm{ng} / \mathrm{ml}$ & $1000 \mathrm{ng} / \mathrm{ml}$ \\
\hline \multicolumn{2}{|l}{ Seuil de détection analytique des amphétaminiques par CPG/SM } & 5 à $10 \mathrm{ng} / \mathrm{ml}$ & - \\
\hline
\end{tabular}

La cocaine est instable sans conservateur du type fluorure de sodium $(\mathrm{NaF})$. En revanche, la benzoylecgonine et l'ecgonine méthylester ses principaux métabolites sont plus stables, même en l'absence d'additif (19).

Les cannabinoïdes sont instables si le contenant est en plastique, mais stables durant des mois si le contenant est en verre ou mieux en verre silanisé (20).

Enfin l'amphétamine et la métamphétamine sont stables dans le sang des mois voire plusieurs années, si le contenant est en verre et hermétiquement clos (21). $\mathrm{Au}$ vu de ces données, il était souhaitable que les prélèvements soient effectués sur tube en verre avec un conservateur comme le fluorure de sodium afin d'éviter au maximum toute dégradation des molécules recherchées et que les analyses soient réalisées dans les plus brefs délais

\section{Fiabilité des méthodes d'identification et de dosa- ge des stupéfiants}

Compte tenu des difficultés analytiques que posent l'identification et le dosage des stupéfiants, la SFTA a recommandé en 1996 des procédures analytiques 
(22-24). Ces méthodes ont largement fait leur preuves puisqu'elles ont été utilisées depuis par les laboratoires qualifiés lors des différents contrôles qualité organisés par la SFTA.

Elles préconisent pour le dosage dans le sang total des cannabinoïdes, des opiacés, des cocaïniques et des amphétaminiques d'utiliser la chromatographie en phase gazeuse couplée à la spectrométrie de masse (CPG/SM) après extraction ainsi que l'utilisation d'étalons internes deutérés.

En effet la CPG/SM est une méthode spécifique et sensible bien adaptée à ces analyses de routine et qui, selon l'arrêté du 5 septembre 2001 fixant les modalités du dépistage des stupéfiants et des analyses prévues par le décret $n^{\circ} 2001-751$ du 27 août 2001, a été choisie comme méthode officielle.

De plus, il est indispensable pour l'expert de garantir la fiabilité de sa méthode de dosage afin d'écarter tout litige sur celle-ci en cas de contre-expertise.

\section{Conclusion}

Cette compilation de 3751 résultats de dosages sanguins, réalisée sur des cas anonymes, basée sur une durée d'un an, a été effectuée par des experts ayant apporté la preuve de leur compétence grâce à des contrôles de qualité externes satisfaisants. Elle confirme la présence de THC et de ses métabolites dans le sang des conducteurs impliqués dans des accidents mortels de la circulation, particulièrement chez les jeunes de moins de 27 ans. Dans cette tranche d'âges, $27,2 \%$ des analyses réalisées étaient positives au $\Delta-9$ THC alors que $2,8 \%$ étaient positives aux opiacés (morphine, codéine, $6 \mathrm{MAM}$ ), 3,8 \% aux amphétaminiques (amphétamine, métamphétamine, MDMA, MDA, MBDB, MDEA) et $1 \%$ aux cocaïniques (cocaïne, benzoylecgonine, méthylecgonine).

Ces chiffres sont cependant sous-évalués par rapport à la réalité pour deux raisons : d'une part en raison du temps écoulé entre l'accident et le prélèvement qui était très souvent supérieur à deux heures et d'autre part car les seuils légaux actuels de positivité sont seion plusieurs auteurs trop élevés, en particulier dans le cas du cannabis pour lequel les taux sanguins usuels sont classiquement bas (de l'ordre de quelques nanogrammes par millilitres) et souvent inférieurs au seuil de positivité légal de $1 \mathrm{ng} / \mathrm{ml}$ de sang.

Une alcoolémie supérieure à $0,5 \mathrm{~g} / \mathrm{l}$ est notée dans environ $20 \%$ des cas positifs au cannabis. Ces deux substances potentialisent leurs effets psychotropes, augmentant les troubles de la vision et altérant l'appréciation des distances, ce qui accroît fortement les risques d'accidents de la circulation.

On observe par ailleurs que la présence des médicaments psychoactifs associés aux stupéfiants est marginale dans le sang des conducteurs impliqués dans un accident mortel de la circulation (environ $5 \%$ sur 448 sangs positifs aux stupéfiants).

Tous ces éléments ainsi que différentes études étrangères (25-28) ont, en France, incité les députés et sénateurs à voter une nouvelle loi, dite loi Dell'Agnola, $\mathrm{n}^{\circ}$ 2003-87 du 3 février 2003 (Journal Officiel du 4 février 2003, p.2103). Cette loi prévoit qu'un OPJ peut faire procéder au dépistage de stupéfiants sur tout conducteur soit impliqué dans un accident mortel ou corporel, ou auteur présumé d'une infraction au code de la route punie d'une suspension du permis de conduire, soit à l'encontre duquel il existe des raisons de soupçonner qu'il a fait usage de stupéfiants. Elle sanctionne la conduite sous l'influence de stupéfiants de deux ans d'emprisonnement et de $4500 €$ d'amende. En cas de présence concomitante de stupéfiants et d'alcool les peines sont portées à trois ans d'emprisonnement et $9000 €$ d'amende. Ces délits donnent également lieu à la réduction de la moitié du nombre de points initial du permis de conduire. Si le dépistage se révèle positif, ou si le conducteur refuse de s'y soumettre, des analyses ou examens cliniques, biologiques ou médicaux sont effectués afin d'établir si la personne conduisait sous l'influence de stupéfiants.

Le décret $n^{\circ} 2003-293$ du 31 mars 2003 paru le $1^{\text {ir }}$ avril 2003 au Journal Officiel stipule trois nouvelles dispositions importantes. Les deux échantillons biologiques prélevés pour la recherche de stupéfiants sont adressés à un expert inscrit sous une rubrique spéciale en toxicologie, sur la liste de la cour d'appel ou à un laboratoire de police technique et scientifique. En cas de retrait, la restitution du permis de conduire se fait après examens médicaux, cliniques et biologiques, notamment capillaires témoignant de l'arrêt de la consommation de stupéfiants. Enfin la recherche de médicaments psychoactifs en cas de positivité aux stupéfiants n'est plus obligatoire.

Ces nouvelles mesures permettront peut-être de réduire le nombre de décès sur les routes de France, pays qui se situe parmi ceux d'Europe où le risque routier est le plus élevé avec près de 8000 tués sur les routes de France par an (soit en 2000, deux fois plus que le Royaume-Uni, la Suède ou les Pays-Bas et une fois et demi plus qu'en Italie) (29). Cette mortalité touche particulièrement les jeunes de 15 à 24 ans, pour qui elle constitue la première cause de mortalité. 


\section{Références}

1. Anger JP. Effets des stupéfiants sur la conduite automobile. Ann. Toxicol. Anal. $2003 ; 15: 71-76$.

2. Mura P., Papet Y., Mauco G. Le risque accidentogène d'une consommation de stupéfiants est-il bien établi ? Ann. Toxicol. Anal. $2003 ; 15: 77-82$.

3. Goullé JP. Effets des stupéfiants chez les conducteurs Législation. Colloque à l'Assemblée Nationale de Paris : «Drogues et Conduite Automobile» - Institut Technique d'Accidentologie. 14 et 15 novembre 2002.

4. Mercier-Guyon C. Le dépistage clinique des conducteurs sous influence de psychotropes. Ann. Toxicol. Anal. 2003 ; $15: 92-97$.

5. Marquet P., Delpla P.A., Kerguelen S. et coll. Prevalence of drugs abuse in urine of drivers involved in road accidents in France : a collaborative study. J. Forensic Sci. $1998 ; 43: 806-811$.

6. Pépin G., Mura P., Kintz P., Dumestre-Toulet V., Ghysel M.H., Goullé J.P., Gruson A., Lhermitte M.A., Lachâtre G., Marka C., Molinaro R., Tourneau J., Vallon J.P. Recherche de stupéfiants dans le sang de conducteurs d'automobiles : résultats d'une compilation française d'expertises toxicologiques. Toxicorama $1999 ; 11: 12-16$.

7. Kintz P., Cirimele V., Mairot F., Muhlmann M., Ludes B. Analyses toxicologiques pratiquées sur 198 conducteurs accidentés. Presse Med. $2000 ; 29: 1275-1278$.

8. Mura P., Kintz P., Ludes B., Gaulier J.M., Marquet P., Martin-Dupont S., Vincent F., Kaddour A., Goullé J.P., Nouveau J., Moulsma M., Tilhet-Coartet S., Pourrat O. Comparison of the prevalence of alcohol, cannabis and other drugs between 900 injured drivers and 900 control subjects : results of a French collaborative study. Forensic Sci. Int. 2003 (sous presse).

9. Mura P. Accidentologie et drogues illicites. Bull. Acad. Natle Méd. 2002, 186, n² , séance du 19 février 2002.

10. Pépin G., Rommel N., Kintz P., Dumestre-Toulet V., Kergueris M.F., Lachatre G., Moulsma M., Goullé J.P., Lacroix C., Ricordel I., Mura P., Vincent F., Gruson A. et A., Lhermitte M., Capolaghi B., Turcant A., Deveaux M., Ghysel M.H., Corteel P. Compilation des résultats de 3751 dosages sanguins de stupéfiants obtenus par 19 experts de laboratoires agréés figurant sur la liste de ceux désignés par le Ministère de l'Intérieur, relative aux analyses toxicologiques de confirmation dans le sang, suite à la parution du décret $n^{\circ} 2001-751$ du 27 août 2001 relatif à la recherche des stupéfiants, pratiquée sur les conducteurs impliqués dans un accident mortel de la circulation. Colloque à l'Assemblée Nationale de Paris : «Drogues et Conduite Automobile» - Institut Technique d'Accidentologie. 14 et 15 novembre 2002.

11. Mura P., Brunet B., Monzon C., Papet Y., Mauco G. Interprétation des concentrations sanguines en cannabinoïdes : données actuelles. Ann. Toxicol. Anal. 2003 ; $15: 35-39$.

12. Deveaux M., Goullé J.P., Lhermitte M. Dosage des stupéfiants dans le sang des conducteurs impliqués dans un accident de la circulation : interprétation des résultats, définition de seuils. Ann. Toxicol. Anal. 2003 ; 15 : 98107.
13. Arditti J., Bourdon J.H., David J.M., Lanza L., Thirion $\mathrm{X}$., Jouglard J. Imprégnation en benzodiazépines de conducteurs impliqués dans des accidents de la circulation. Presse Med. 1993 ; 22 : 765-766.

14. Oster G., Huse D.M., Adams S.F., Imbimbo J., Russell M.W. Benzodiazepine tranquillizers and the risk of accidental injury. Am. J. Public Health 1990 ; 80 : 1467-1470.

15. Oster G., Russell M.W., Huse D.M., Adams S.F., Imbimbo J. Accident and Injury related health care utilization among benzodiazepine users and nonusers. J. Clin. Psychiatry $1987 ; 48$ (suppl) : 17-21.

16. Hemmelgarn B., Suissa S., Huang A., Boivin J.F., Pinard G. Benzodiazepine use and the risk of motor vehicle crash in the elderly. JAMA $1997 ; 278: 27-31$.

17. Ray W.A., Fought R.L., Decker M.D. Psychoactive drugs and the risk of injurious motor vehicle crashes in elderly drivers. Am. J. Epidemiol. $1992 ; 136$ : 873-883.

18. Dumestre-Toulet V., Verstraete A. Les adultérants des tests urinaires. Toxicorama $1999 ; 3: 155-166$.

19. Moody D.E., Monti K.M., Spanbauer A.C. Long term stability of abused drugs and antiabuse chemotherapeutical agents stored at $-20^{\circ}$ C. J. Anal. Toxicol. 1999 ; $23(6): 535-540$.

20. Johnson J.R., Jennison T.A., Peat M.A., Foltz R.I. Stability of delta-9-THC, 11-OH-THC and 11-nor-9-carboxy-THC in blood and plasma. J. Anal. Toxicol. 1984 ; $8(5)$ : 202-204.

21.Nagata T., Kimura K., Hara K., Kudo K. Methamphetamine and amphetamine concentrations in post mortem rabbit tissues. Forensic Sci. Int. 1990 ; 48 : 39-47.

22. Kintz P., Cirimèle V., Pépin G., Marquet P., Deveaux M., Mura P. Identification et dosage des cannabinoïdes dans le sang total. Toxicorama $1996 ; 8: 29-33$.

23. Marquet P., Lachâtre G., Kintz P., Pépin G., Deveaux M., Mura P. Identification et dosage des principales drogues amphétaminiques dans le sang total par chromatographie en phase gazeuse couplée à la spectrométrie de masse (CPG-SM). Toxicorama 1996 ; $8: 23-28$.

24. Gaillard Y., Pépin G., Marquet P., Kintz P.. Deveaux M., Mura P. Identification et dosage de la benzoylecgonine, cocaïne, méthylecgonine-ester, codéine, morphine et 6acétylmorphine dans le sang total. Toxicorama 1996; 8 : 17-22.

25. Verstraete A. Les actions réalisées dans les autres pays. Colloque à l'Assemblée Nationale de Paris : «Drogues et Conduite Automobile» - Institut Technique d'Accidentologie. 14 et 15 novembre 2002.

26. Dussault C., Brault M., Bouchard J., Lemire A.M. Le rôle de l'alcool et des autres drogues dans les accidents mortels de la route au Quebec - Résultats préliminaires. Colloque à l'Assemblée Nationale de Paris : «Drogues et Conduite Automobile» - Institut Technique d'Accidentologie. 14 et 15 novembre 2002.

27. Augsburger M. Stupéfiants et conduite automobile - les actions réalisées en Suisse. Ann. Toxicol. Anal. 2003 ; $15: 138-144$.

28. Moeller M. Stupéfiants et conduite automobile - les actions réalisées en Allemagne. Ann. Toxicol. Anal. 2003 ; 15 : 145-152.

29. Transport 2000. Evolution des transports, CEMT, Paris, 2002 ; BICAR. 